\title{
Immunological and clinical immunotherapy implications of NLRP3 mutations in melanoma
}

\author{
Qinghua Wang ${ }^{1,}{ }^{*}$, Juncheng Lyu ${ }^{1,}{ }^{*}$, Wenjing Zhang ${ }^{1}$, Fuyan Shi ${ }^{1}$, Yanfeng Ren ${ }^{1}$, Qian Mao ${ }^{1}$, \\ Yujie Liu' ${ }^{1}$, Yuting Li ${ }^{2}$, Suzhen Wang ${ }^{1}$ \\ ${ }^{1}$ Department of Health Statistics, Key Laboratory of Medicine and Health of Shandong Province, School of Public \\ Health, Weifang Medical University, Weifang 261053, Shandong, China \\ ${ }^{2}$ Tianjin Cancer Institute, National Clinical Research Center for Cancer, Key Laboratory of Cancer Prevention and \\ Therapy of Tianjin, Tianjin Medical University Cancer Institute and Hospital, Tianjin 300060, Tianjin, China \\ *Equal contribution
}

Correspondence to: Suzhen Wang; email: wangsz@wfmc.edu.cn

Keywords: melanoma, immunotherapy, NLRP3 mutations, predictive indicator, clinical practice

Received: June 17, 2021

Accepted: August 24, 2021

Published: November 8, 2021

Copyright: (C) 2021 Wang et al. This is an open access article distributed under the terms of the Creative Commons Attribution License (CC BY 3.0), which permits unrestricted use, distribution, and reproduction in any medium, provided the original author and source are credited.

\section{ABSTRACT}

Recent studies have demonstrated the role of Nod-like receptor protein 3 (NLRP3) inflammasome in promoting melanoma progression. Immune checkpoint inhibitors (ICI) treatment dramatically extended the survival outcomes for advanced melanoma patients. Nevertheless, immunologic and immunotherapy implications of NLRP3 mutations in melanoma were obscure. Herein, we utilized publicly genomic data of 750 melanoma patients to explore the association of NLRP3 mutations with immunologic and genomic features. In addition, we curated 336 advanced/metastatic melanoma patients treated with ICl agents from 6 published studies to analyze the response rate and survival outcome in relation to NLRP3 mutations. We observed that patients with NLRP3 mutations had a significantly higher tumor mutation burden $(P<0.001)$ and neoantigen burden $(P<$ 0.001). Moreover, significantly lower tumor heterogeneity $(P=0.048)$ and purity $(P=0.022)$ were also observed in this mutated subgroup. Elevated infiltration of immune-response cells, decreased enrichment of immunesuppressive cells, and immune response-related circuits were markedly enriched in patients with NLRP3 mutations. In the pooled ICl-treated cohort, NLRP3 mutations were linked with the higher response rate $(P=$ $0.031)$ and preferable survival outcome $(P=0.006)$. NLRP3 mutations were identified to associate with the elevated mutational burden, favorable immune infiltration, and preferable ICl efficacy. Findings derived from our study suggest that NLRP3 mutations may serve as a potential biomarker for evaluating melanoma immunotherapy response.

\section{INTRODUCTION}

Due to the evidently better variability in immunogenicity during tumor progression, melanoma is broadly considered as an immunogenic malignancy [1], which serves as the model system for evaluating the effectiveness of invented immunotherapies [2]. Therefore, therapies for melanoma have been recently changed owing to the emergence of immune checkpoint inhibitors (ICI) including anti-CTLA-4 and anti-PD-1 agents [3]. ICI therapies have observably extended the survival time for advanced melanoma patients $[4,5]$. Nevertheless, the remarkable efficacy was only observed in a fraction of patients, most were not benefitted.

Recent studies demonstrated that ICI therapies were influenced by a combination of predictive biomarkers related to genomics, immune checkpoints expression, characteristics of the microenvironment, and gut 
microbiome [6]. Tumor mutation burden (TMB) and neoantigen burden (NB) emerged as promising markers for evaluating ICI efficacy and previous findings have demonstrated their positive association with the immunotherapy response rate and prognosis via numerous clinical trials [7-9]. However, a few studies concluded controversial results, that is high TMB sometimes could not accurately predict ICI response [8]. Immune checkpoints, such as programmed cell death ligand 1 (PD-L1) expression is another widely used biomarker associated with ICI therapies efficacy. Similarly, it may be out of work in some trials [10]. In view of the current situation, novel and more effective indicators were needed to distinguish subpopulations that are likely to be sensitive to ICI treatment.

Nod-like receptor protein 3 (NLRP3) inflammasome was a three-domain complex associated with inflammation regulation, immune response, and cell apoptosis [11]. Functions of NLRP3 inflammasome in cancer progression remained inconsistent owing to the controversial results reported [12]. For instance, the protective effects of this inflammasome were observed in colon cancer $[13,14]$. Conversely, it exhibited a promotion role in cancers of gastric [15], liver [16], head and neck [17], lung [18], prostate [19], glioblastoma [20], and melanoma [21].

Recent studies demonstrated that NLRP3 inflammasome upregulation may inhibit the inflammatory responses in melanoma. Consistently, a mice model with NLRP3 deficiency showed the protection roles against cancer progression $[22,23]$. The progression of cancer cells could be suppressed by reduced NLRP3 inflammasome and IL-1 $\beta$ expression [24]. It has been shown that NLRP3 downregulation and reduced IL- $1 \beta$ secretion decreased metastatic melanoma by thymoquinone therapy in a mouse model [21]. Evaluation of the roles of NLRP3 inflammasome in the immune response by employing vaccination against the melanoma cells demonstrated that mice with NLRP3 vaccination deficiency who received a subcutaneous injection of poorly immunogenic melanoma cells leading to a 4-fold promotion in survival times as compared to the control mice [25]. NLRP3 plays vital roles in melanoma tumorigenesis, progression, and immune response, however, its alterations association with ICI efficacy remains unclear.

Herein, we analyzed whether NLRP3 mutations were correlated with immunological and genomic features with publicly available data in melanoma. Further, the association of NLRP3 mutations with ICI efficacy was estimated with an aggregated ICI-treated cohort. Novel findings would provide implications for tailoring clinical trials and immunotherapeutic strategies for melanoma.

\section{RESULTS}

\section{NLRP3 mutations in melanoma}

In the TCGA cohort, $89(19.1 \%)$ of 467 melanoma patients harbored NLRP3 mutations. NLRP3 is one frequently mutated gene and we found that patients with NLRP3 mutations had higher TML as compared with others (Figure 1A). Of 89 NLRP3 mutated patients, 53 $(59.5 \%)$ also had mutations of genes related to genomic maintenance including TP53, BRCA1/2, POLE, and MMR genes (Figure 1B). Further analyses revealed that NLRP3 mutated patients harbored significantly higher mutation rates of above genome repair genes than $N L R P 3$ wild-type patients (Fisher exact test, all $P<$ 0.05 ; Figure 1C). Mutational patterns of NLRP3, its family members, and genomic maintenance genes were exhibited in Figure 1B.

\section{NLRP3 mutations were correlated with high TMB, $\mathrm{NB}$, and favorable genomic features}

In the TCGA cohort, patients with NLRP3 mutations had significantly higher TMB than patients without (Wilcoxon rank-sum test, $P<0.001$; Figure 2A). We observed that genome repair regulators including BRCA1/2, TP53, POLE, and MMR genes were frequently mutated and mutations of these genes also induced significantly higher TMB (all $P<0.01$; Figure 2B). Multivariate Logistic regression model included clinical variables (i.e., age, gender, and stage) and mutations of above genome repair genes was performed to adjust confounding factors. Association of NLRP3 mutations with higher TMB was still significant (OR: 7.50, 95\% CI: 3.85-15.24, $P<0.001$; Figure 2B). In 2 independent cohorts from ICGC, the Wilcoxon ranksum test showed that NLRP3 mutated patients also had significantly higher TMB than NLRP3 wild-type patients (both $P<0.001$; Supplementary Figure 1A, 1C). Further multivariate regression model obtained consistent findings after adjusting clinical and genomic confounders (MELA-AU cohort [OR: 8.87, 95\% CI: 3.04-29.63, $P<0.001$ ]; SKCA-BR cohort [OR: 10.38, 95\% CI: 1.38-101.34, $P=0.028$ ]; Supplementary Figure 1B, 1D).

Significantly elevated NB was observed in patients with NLRP3 mutations (Wilcoxon rank-sum test, $P<0.001$; Figure 2C). Multivariate Logistic model with other confounders taken into account remained statistically significant (OR: 3.94, 95\% CI: 1.82-9.04, $P<0.001$; Figure 2D).

Lower tumor heterogeneity and purity, which suggest the lower proportions of subclonal mutations and tumor cells in microenvironment, were statistically associated 
with NLRP3 mutations (Wilcoxon rank-sum test, $P=$ 0.048 and $P=0.022$; Figure $2 \mathrm{E}, 2 \mathrm{~F}$ ). No significant difference was found in tumor ploidy based on NLRP3 mutational statuses (Wilcoxon rank-sum test, $P=0.49$; Figure 2G). The above findings demonstrated that $N L R P 3$ mutations were linked with favorable genomic characteristics.

\section{NLRP3 mutations were correlated with the better microenvironment}

ESTIMATE algorithm showed that the difference of overall immune cells infiltration was not significant between NLRP3 mutated and wild-type patients (Wilcoxon rank-sum test, $P=0.329$; Supplementary
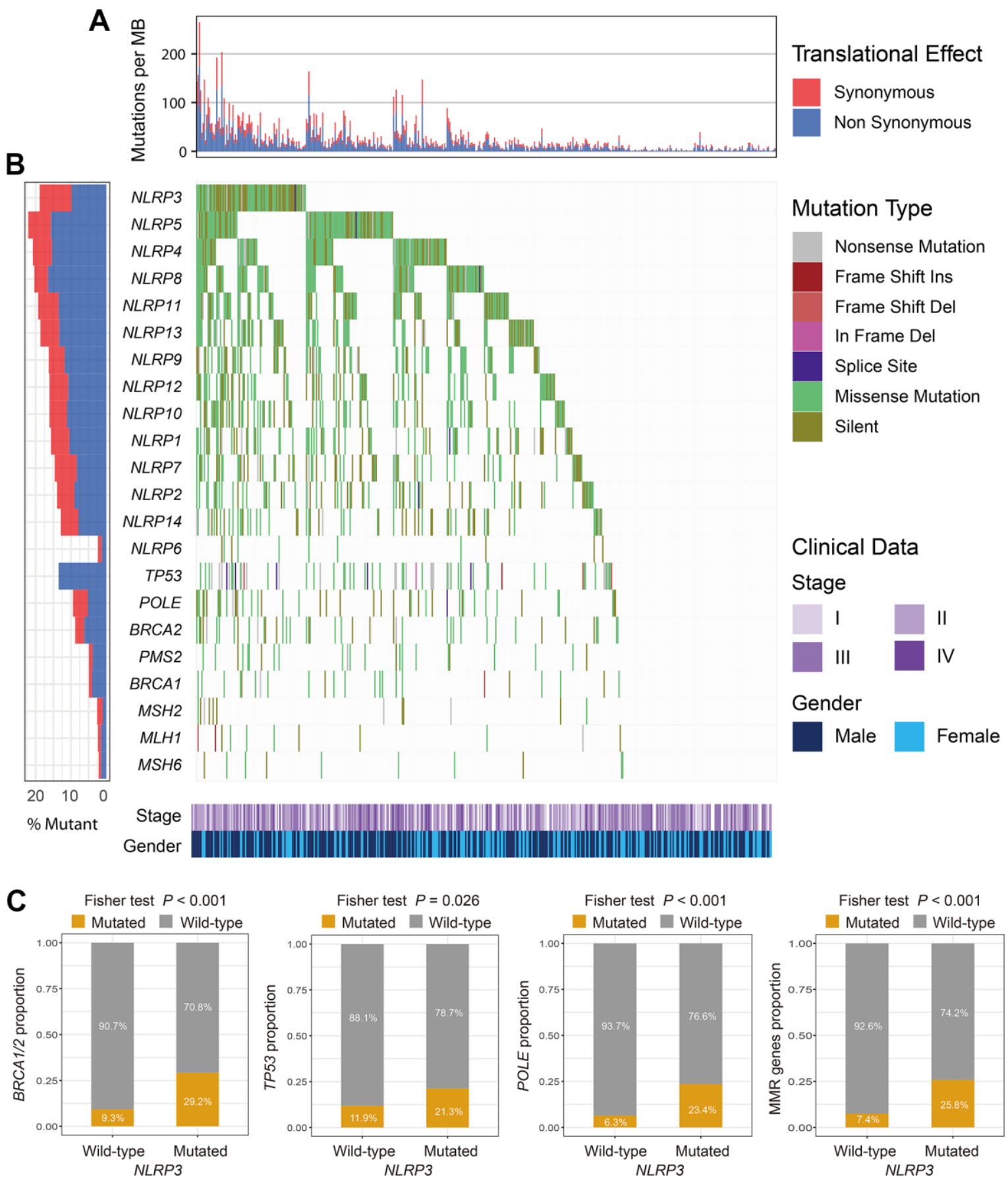

Figure 1. The mutational patterns of NLR family members and genome maintenance genes. (A) TMB stratified by synonymous and non-synonymous mutations for each patient. (B) Waterfall plot for NLR family members and genome maintenance genes. (C) Association of NLRP3 mutations with BRCA1/2, TP53, POLE, and MMR genes mutations. 
Figure 2). We thus estimated the abundance of distinct immune cell subtypes using the CIBERSORT approach and compared their differences based on NLRP3 statuses. Results revealed that significantly lower
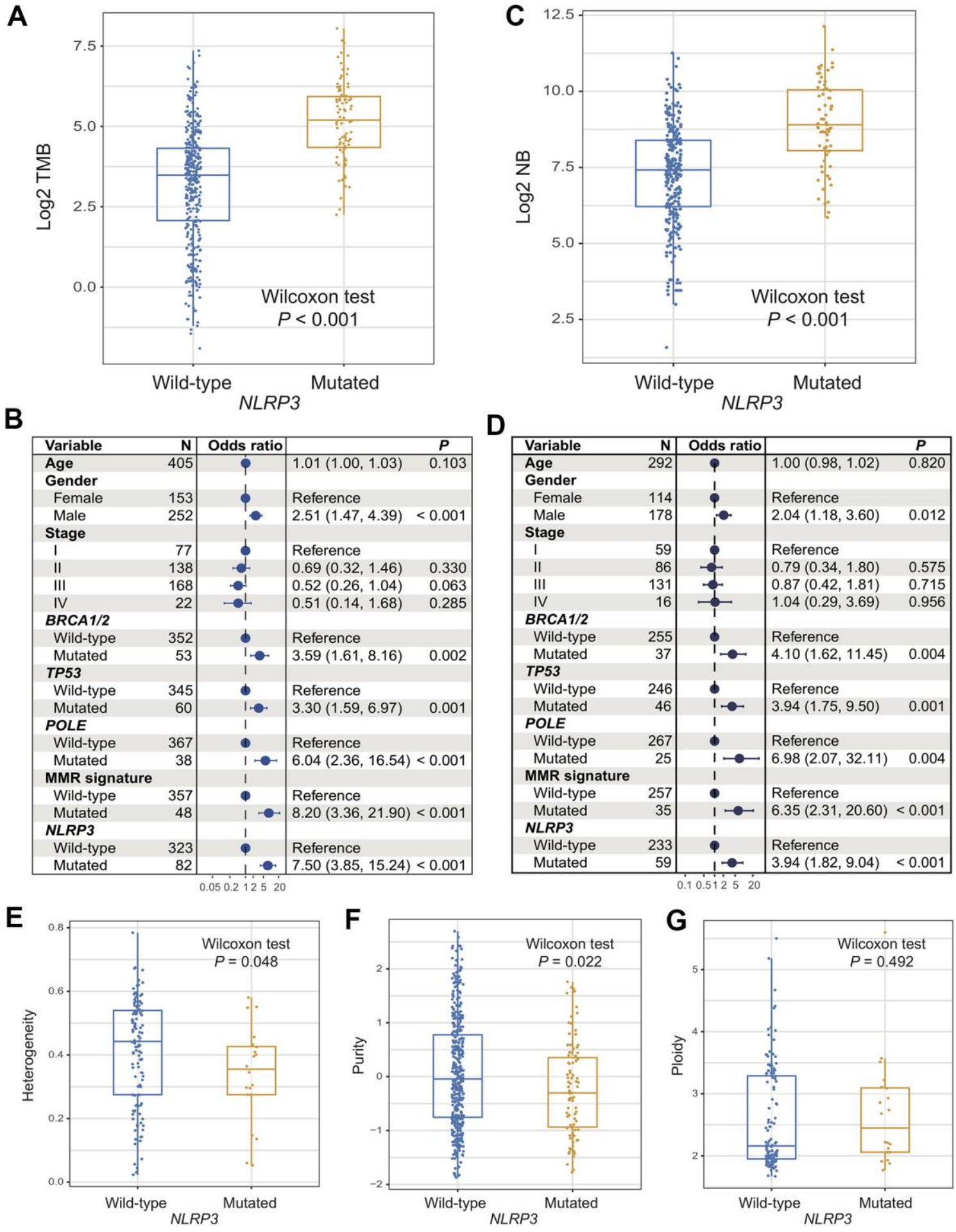

Figure 2. Association of NLRP3 mutations with TMB, NB, and genomic features. (A, B) NLRP3 mutations versus TMB with univariate analysis and multivariate regression model. (C, D) NLRP3 mutations versus NB with univariate analysis and multivariate regression model. NLRP3 mutations association with (E) tumor heterogeneity, (F) purity, and (G) ploidy. 
A

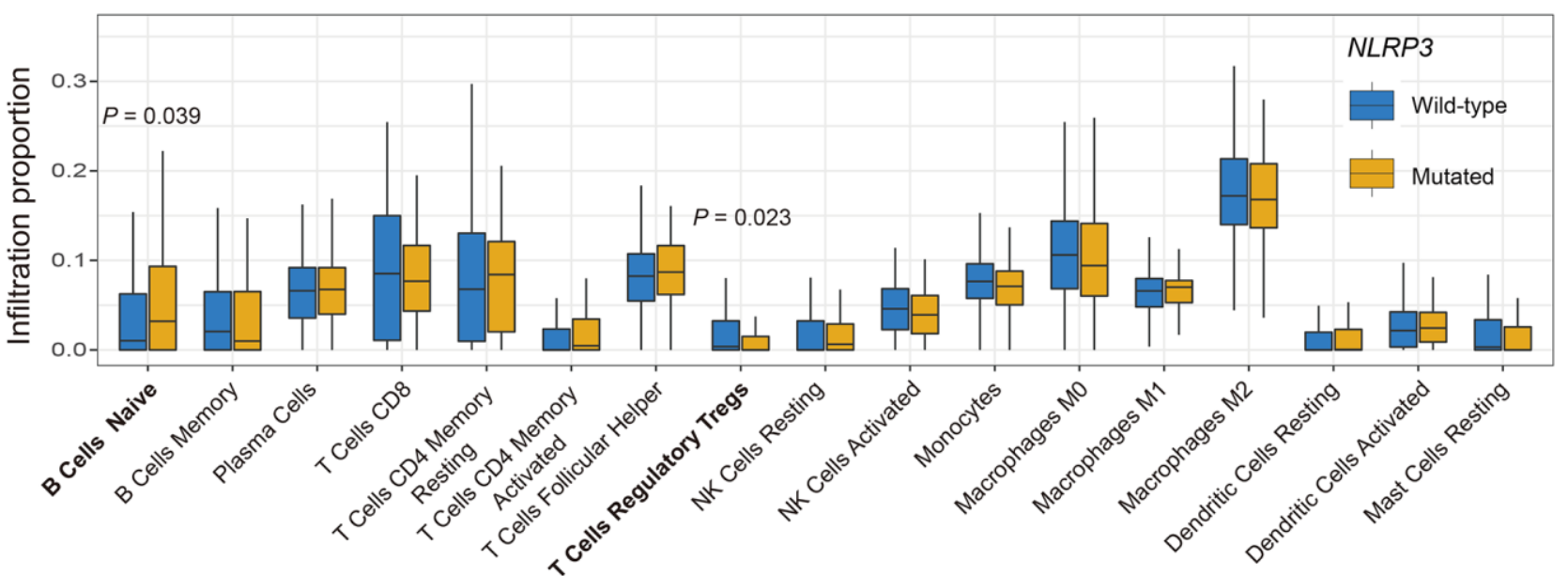

B

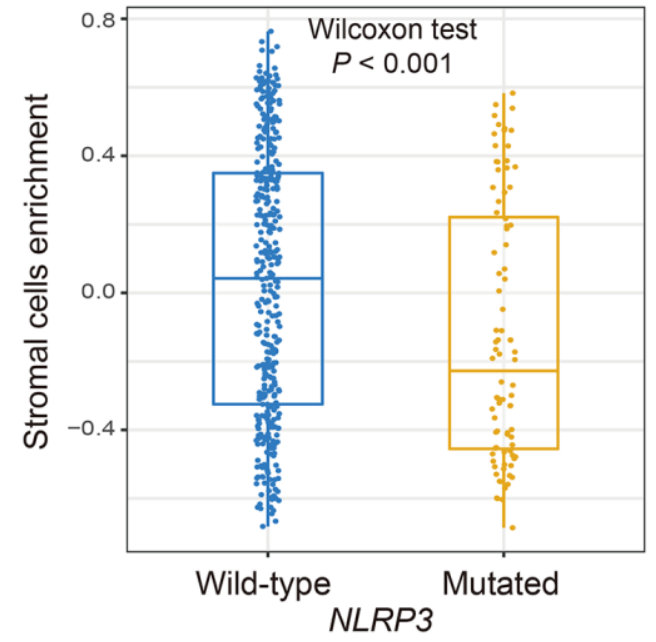

C

\begin{tabular}{|c|c|c|c|c|}
\hline Variable & $\mathbf{N}$ & Odds ratio & & $P$ \\
\hline Age & 403 & (1) & $0.99(0.97,1.00)$ & 0.06 \\
\hline Gender & & & & \\
\hline Female & 152 & & Reference & \\
\hline Male & 251 & & $0.85(0.56,1.30)$ & 0.45 \\
\hline \multicolumn{5}{|l|}{ Stage } \\
\hline I & 76 & & Reference & \\
\hline II & 138 & & $0.55(0.29,1.00)$ & 0.05 \\
\hline III & 167 & & $1.23(0.70,2.17)$ & 0.47 \\
\hline IV & 22 & & $1.91(0.70,5.67)$ & 0.22 \\
\hline \multicolumn{5}{|l|}{ BRCA1/2 } \\
\hline Wild-type & 350 & & Reference & \\
\hline Mutated & 53 & & $0.74(0.39,1.41)$ & 0.37 \\
\hline \multicolumn{5}{|l|}{ TP53 } \\
\hline Wild-type & 344 & & Reference & \\
\hline Mutated & 59 & & $0.86(0.47,1.57)$ & 0.63 \\
\hline POLE & & I & & \\
\hline Wild-type & 366 & 甲 & Reference & \\
\hline Mutated & 37 & 一 & $1.97(0.93,4.30)$ & 0.08 \\
\hline \multicolumn{5}{|c|}{ MMR signature } \\
\hline Wild-type & 356 & & Reference & \\
\hline Mutated & 47 & & $0.95(0.48,1.84)$ & 0.87 \\
\hline \multicolumn{5}{|l|}{ NLRP3 } \\
\hline Wild-type & 322 & (1) & Reference & \\
\hline Mutated & 81 & -1 & $0.53(0.30,0.92)$ & 0.03 \\
\hline
\end{tabular}

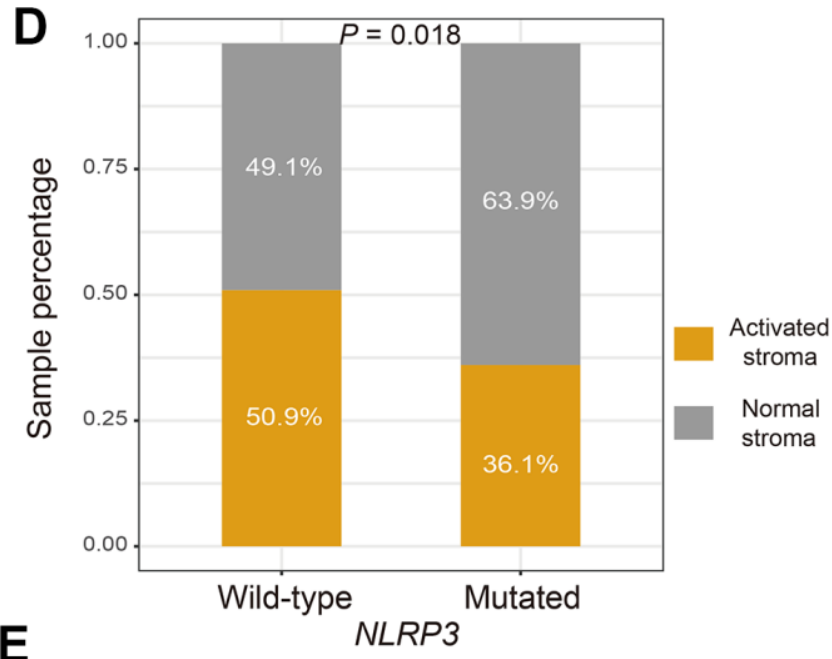

E

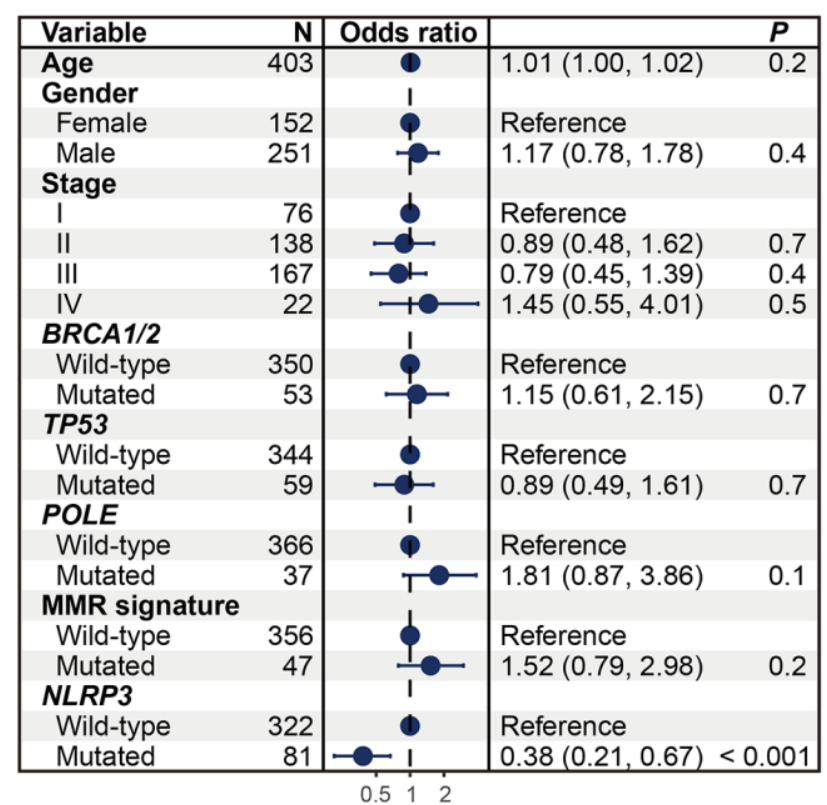

Figure 3. Association between NLRP3 mutations and factors in microenvironment. (A) Diverse infiltration abundance of immune cells based on NLRP3 mutational status. (B) Differential enrichment of overall stromal cells in NLRP3 mutated and wild-type patients. (C) Representation for forest plot of association between NLRP3 mutations and stromal cells enrichment. (D) Distinct distribution of activated stroma subtype in patients with and without NLRP3 mutations. (E) Multivariate Logistic regression model for the association of NLRP3 mutations with activated stroma subtype. 
immune cells infiltration according to NLRP3 mutational statuses via the Angelova et al. method. Consistent with the result of CIBERSORT, significantly lower infiltration of regulatory $\mathrm{T}$ cells was also found in patients with NLRP3 mutations (Wilcoxon rank-sum test, $P=0.045$; Supplementary Figure 3 ). In addition, we observed a higher abundance of activated CD4 T cells, effector CD4 T cells, and dendritic cells in NLRP3 mutant patients (Wilcoxon rank-sum test, all $P<0.05$; Supplementary Figure 3).

Stromal cells could promote tumor growth and inhibit immune response. Results indicated that patients with NLRP3 mutations harbored significantly lower enrichment of overall stromal cells (Wilcoxon rank-sum test, $P<0.001$; Figure 3B). Multivariate Logistic regression model with other confounding factors taken into account still remained significant (OR: 0.53, 95\% CI: $0.30-0.92, P=0.03$; Figure $3 \mathrm{C}$ ). Proportion of activated stroma subtypes was significantly decreased in NLRP3 mutated patients as compared with wild-type patients (proportion: $36.1 \%$ vs. $50.9 \%$, Fisher exact test, $P=0.018$; Figure 3D). This result was more significant in multivariate analysis (OR: $0.38,95 \%$ CI: $0.21-0.67$, $P<0.001$; Figure 3E).

Differential analyses of immune checkpoint genes showed that only CD276 exhibited a significantly elevated expression in NLRP3 mutated patients (Wilcoxon rank-sum test, $P=0.024$; Supplementary Figure 4). Other checkpoint genes did not show statistical significance (Wilcoxon rank-sum test, all $P$ > 0.05; Supplementary Figure 4). Collectively, the activated immune microenvironment was enriched in melanoma patients with NLRP3 mutations.

\section{Immune response pathways correlated with $N L R P 3$ mutations}

Results of GSEA analysis demonstrated that immune response-related signaling pathways, including graft versus host disease (normalized enrichment score: 2.02, $F D R=0.007$; Supplementary Figure 5) and allograft rejection (normalized enrichment score: 1.74, FDR = 0.024; Supplementary Figure 5) were significantly enriched in the top circuits of NLRP3 mutations.

\section{Clinical characteristics versus ICI efficacy in immunotherapy cohort}

Before evaluating the association of NLRP3 mutations with ICI efficacy, we explored the influences of common clinical features (i.e., TMB, age, gender, stage, and treatment type) with 336 ICI-treated melanoma patients. We observed that high TMB was associated with elevated response rate (response rate: $35.7 \%$ vs.
$25.0 \%$, Fisher exact test, $P=0.043$ ) and preferable overall survival (OS) (Log-rank test, $P=0.049$ ) (Supplementary Figure 6A). Patients with age $>60$ were likely to have a higher response rate than others (response rate: $41.4 \%$ vs. $23.7 \%$, Fisher exact test, $P=$ 0.002), but they did not exhibit a statistical difference in prognosis (Log-rank test, $P=0.942$ ) (Supplementary Figure 6B). Male patients harbored a trend of high response rate, although it did not reach the statistical significance (response rate: $35.9 \%$ vs. $25.3 \%$, Fisher exact test, $P=0.054$ ); there is no significant difference in OS (Log-rank test, $P=0.286$ ) (Supplementary Figure 6C). Patients with advanced-stage had the lowest response rate and worst prognosis, which may be correlated with their intrinsic properties (Supplementary Figure 6D). We found patients treated with anti-PD-1 therapy had the highest response rate than patients treated with anti-CTLA-4 or combined therapy (response rate: $41.6 \%$ vs. $31.8 \%$ vs. $16.3 \%$, Fisher exact test, $P=0.002$ ); nevertheless, patients who received combined therapy had the best OS (Log-rank test, $P=$ 0.044) (Supplementary Figure 6E).

\section{NLRP3 mutations were linked with favorable ICI efficacy}

Associations of NLRP3 mutations with clinical characteristics among 336 ICI-treated melanoma patients were exhibited in Supplementary Table 1. Consistent with the aforementioned result, significantly increased TMB was identified in patients with NLRP3 mutations in the immunotherapy cohort (Wilcoxon rank-sum test, $P<0.001$; Supplementary Figure 7A, 7B).

We found that NLRP3 mutated patients had significantly higher response rate than wild-type patients (response rate: $45.2 \%$ vs. $28.2 \%$, Fisher exact test, $P=0.031$; Figure 4A). Multivariate Logistic regression model also showed statistical difference after adjusting confounders (i.e., age, gender, stage, ICI therapy type, and TMB) (OR: $0.59,95 \%$ CI: $0.28-1.25$, $P=0.095$; Figure 4B).

We calculated the intrinsic prognostic ability of NLRP3 mutations using 3 ICI-treated-naive cohorts from TCGA and ICGC. Results showed that NLRP3 mutations were not correlated with prognoses (Log-rank test, $P=0.461$, $P=0.686$ and $P=0.916$; Supplementary Figure 8). However, survival analysis suggested that NLRP3 mutated patients had significantly preferable overall survival than wild-type patients in the ICI-treated cohort (median OS: not available [because more than half the patients in this subgroup were alive] vs. 20.9 [95\% CI, 16.9-24.9], Log-rank test, $P=0.006$; Figure 4C). Multivariate Cox regression analysis with clinical 
variables taken into account still remained significant (HR: 0.53, 95\% CI: 0.31-0.91, $P=0.021$; Figure 4D).

Finally, associations of NLRP3 mutations with ICI efficacy in distinct therapies were explored respectively. In patients treated with anti-CTLA-4 agents, no association was found between NLRP3 mutations and response rate (response rate: $38.1 \%$ vs. $31.1 \%$, Fisher exact test, $P=0.685$; Figure 5A); however, NLRP3 mutations were associated with better OS, although this result did not obtain statistical significance (Log-rank test, $P=0.063$; Figure $5 \mathrm{~B}$ ). In patients treated with antiPD-1 agents, we found that patients with NLRP3
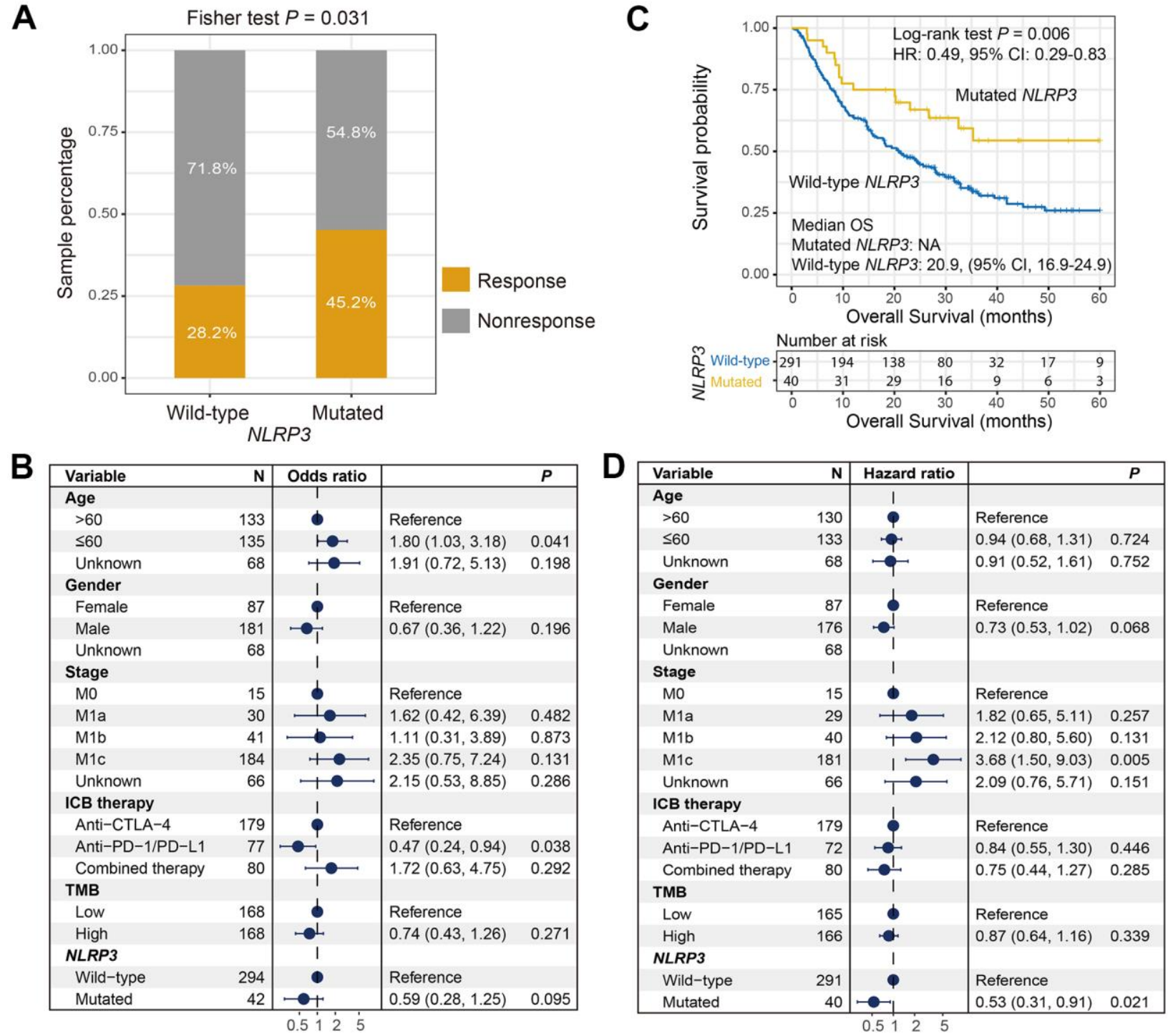

Figure 4. Correlation of NLRP3 mutations with ICI response rate and survival interval. (A) Distinct ICI response rate in NLRP3 mutated and wild-type patients. (B) Association of NLRP3 mutations with the response rate in multivariate Logistic regression model. (C) Kaplan-Meier survival curve of distinct NLRP3 status in ICl-treated cohort. (D) Forest plot for multivariate Cox regression model with confounders taken into account. 


\section{DISCUSSION}

By integrating and analyzing available genomic and clinical data of melanoma, NLRP3 mutations were identified to be associated with higher mutation and neoantigen burden, favorable microenvironment, and better tumor genomic features. Importantly, our study demonstrated that the elevated response and prolonged survival time of ICI therapy were found in patients with NLRP 3 mutations. These findings suggest the predictive implications of NLRP3 mutations for melanoma immunotherapy.

Previously many studies revealed the vital roles of mutations of a single gene in evaluating ICI therapy efficacy. Jia et al. found that TTN mutations were positively associated with ICI predictive biomarkers and immunotherapy survival interval in melanoma and nonsmall cell lung cancer [26]. Patients with POLE/POLDI mutations exhibited a significantly preferable prognosis in a multi-cancer-ICI cohort with 1644 patients [27]. In metastatic renal cell carcinoma patients who received Nivolumab antibody, Braun et al. observed that favorable overall and progression-free survival were markedly correlated with PBRMI mutations [28]. High TMB and NB are 2 promising biomarkers in cancer immune treatment, however, some factors such as uncertain threshold, exome sequencing fees, and bias of different platforms largely influence the precise evaluation for both markers [26]. Mutations of NLRP3 could accurately assess high TMB and NB as our results described. Therefore, instead of choosing TMB and NB, $N L R P 3$ mutations may be an alternative surrogate for predicting ICI response in melanoma.

Low tumor heterogeneity and purity suggest the reduced proportion of subclonal mutations and tumor cells in the microenvironment, which were reported to be correlated with better response to anti-PD-1 therapy [29]. Our study found that patients with NLRP3 mutations had decreased heterogeneity and purity, indicated the vital roles of NLRP3 mutations in
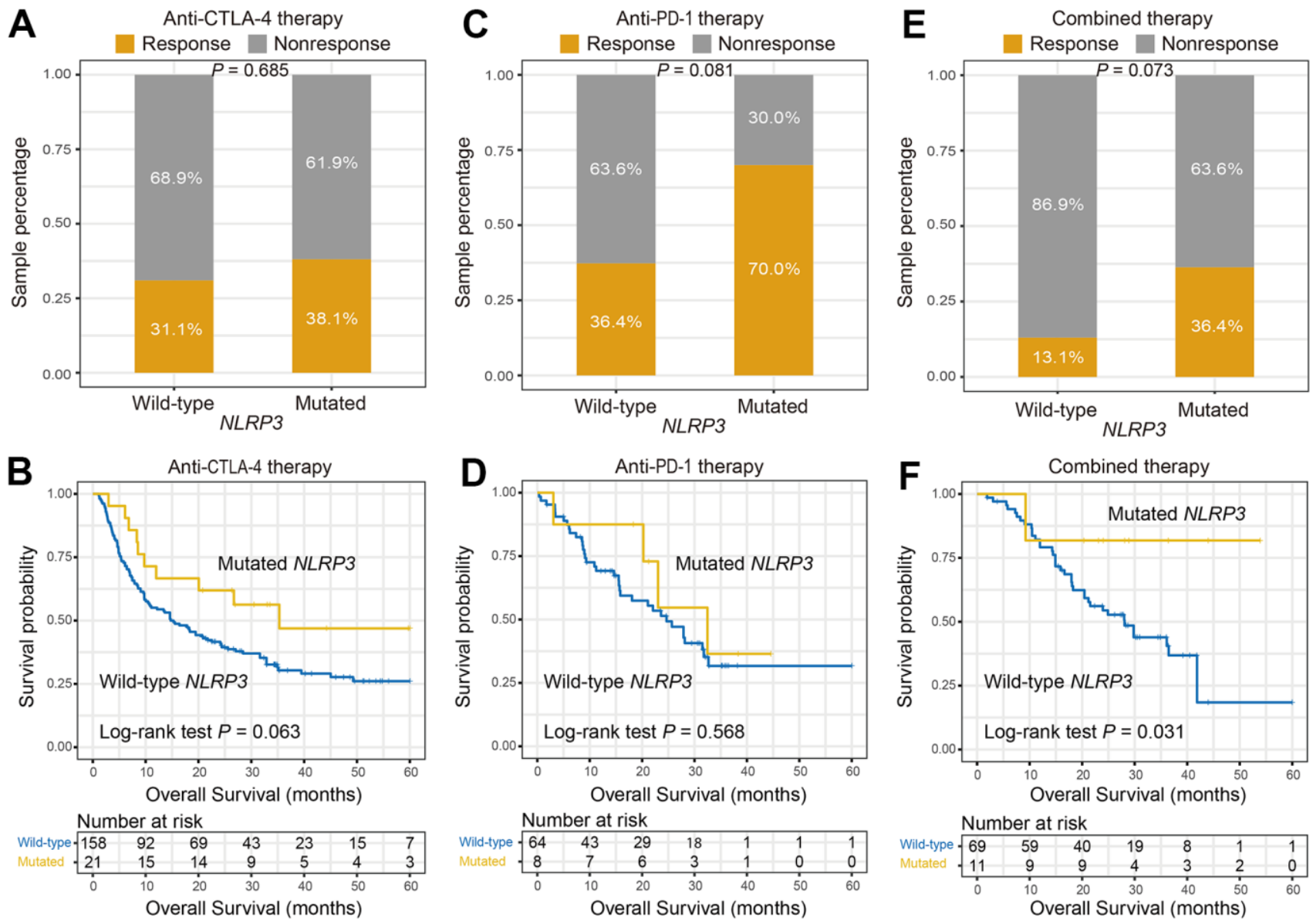

Figure 5. NLRP3 mutations association with ICl efficacy in distinct therapies. (A, B) Association of NLRP3 mutations with response rate and prognosis in patients treated with anti-CTLA-4 agents. (C, D) NLRP3 mutations versus response rate and prognosis in patients treated with anti-PD-1 agents. (E, F) NLRP3 mutations versus response rate and prognosis in patients who received combined therapy. 
immunotherapy response. The regulatory $\mathrm{T}$ cell is one immune cell subtype that performed immunesuppressive roles as well as stromal cells [30, 31]. NLRP3 mutations were found to be correlated with lower infiltration of regulatory $\mathrm{T}$ cells and stromal cells. Moreover, NLRP3 mutant patients harbored a reduced proportion of activated stroma subtype, which exhibits the roles of immune suppression. The above findings further verify the potentially positive association behind $N L R P 3$ mutations and ICI therapy response.

In non-ICI-treated cohorts, no survival benefits were found in patients with NLRP3 mutations. Nevertheless, NLRP3 mutations exhibited a preferable response rate and ICI efficacy in the aggregated ICI cohort. Noticeably, for the roles of NLRP3 mutations in specific therapy, we found that $N L R P 3$ mutated patients could obtain the best survival benefit during combined therapy as compared with anti-CTLA-4 and anti-PD-1 therapies. These findings suggest that NLRP3 mutations may serve as a predictive indicator for evaluating the efficacy of ICI, especially combined immunotherapy.

Recent research demonstrated that reduced NLRP3 inflammasome and IL-1 $\beta$ expression could inhibit the progression of cancer cells [24]. Consistently, another study reported that NLRP3 downregulation and reduced IL-1 $\beta$ secretion decreased metastatic melanoma by thymoquinone therapy in a mouse model [21]. The above findings suggested the crucial roles of NLRP3 and IL-1 $\beta$ in tumorigenesis and the development of melanoma. In this work, we also evaluated the markedly positive association of $N L R P 3$ and $I L-1 \beta$ expression in melanoma (Supplementary Figure 9A) and further confirmed the collective roles of both regulators. Nevertheless, no significant differences were detected in NLRP3 wild-type and mutated subgroups with respect to $N L R P 3$ and $I L-1 \beta$ expression (Supplementary Figure 9B, 9C). These results suggested that the mechanisms underlying the association between NLRP3 mutation and favorable immunotherapy efficacy may neither involve in $N L R P 3$ nor $I L-1 \beta$ transcriptional signals.

A few limitations existed. Firstly, the gene expressionrelated analyses were performed with only the TCGA cohort, no additionally available datasets were used for validation. Secondly, biological relevance between $N L R P 3$ mutations and immunological features was elusive, further studies were needed to explore.

In melanoma, NLRP3 mutations were associated with better immunological and genomic characteristics. It is worth noting that the elevated response rate and favorable ICI survival were also observed in NLRP3 mutated patients. NLRP3 mutations may harbor vitally predictive implications for immunotherapy response in melanoma.

\section{MATERIALS AND METHODS}

\section{Somatic mutation data, gene expression profile, and clinical information of included melanoma patients}

Somatic mutation data of 467 melanoma patients in the Cancer Genome Atlas (TCGA) were derived from Genome Data Commons (https://portal.gdc.cancer.gov). MELA-AU and SKCA-BR cohorts respectively containing 183 and 100 patients derived from the International Cancer Genome Consortium (ICGC) (https://dcc.icgc.org) were utilized for specific validation. Gene expression data of 465 patients were acquired from the TCGA cohort.

From previously published 6 studies $[1,32-36]$, we collected a total of 336 pre-treatment advanced/ metastatic melanoma patients with somatic mutation data. These patients were treated with anti-PD-1 agents, anti-CTLA-4 agents, or combined therapy in their trials. All somatic mutations were uniformly annotated with Oncotator [37]. Clinical information (e.g., age, gender, stage, and treatment type) and ICI efficacy (i.e., response status and survival time) of these 336 patients were described in Supplementary Table 2. In this study, patients with statuses of completed or partial response were defined as responders, other statuses including stable and progressive disease were not considered to be efficacious to ICI therapy.

\section{Association of NLRP3 mutations with TMB, NB, and genomic features}

Mutations of genomic maintenance genes were largely correlated with genome instability. Therefore, in addition to univariate analysis of the association of NLRP3 mutations with $\mathrm{TMB}$ and $\mathrm{NB}$, we also conducted a multivariate Logistic regression model with mutations of DNA damage repair genes (i.e., TP53, $B R C A 1 / 2$, and $P O L E$ ) and mismatch repair (MMR) genes (i.e., $M L H 1, M S H 2, M S H 6$, and $P M S 2$ ) taken into account to control false positive. The neoantigen data of 340 patients were acquired from the Cancer Immunome Atlas (TCIA, https://www.tcia.at/home). TMB and NB were stratified into high and low subgroups with the median.

A number of studies have reported the vital roles of tumor genomic features (i.e., heterogeneity, purity, and ploidy) on immune response and immunotherapeutic efficacy $[29,38]$. We therefore utilized relevant data of 140 patients derived from TCIA to evaluate the association of NLRP3 mutations with heterogeneity and 
ploidy. For the tumor purity of each patient, we used the ESTIMATE algorithm embedded in $\mathrm{R}$ package ESTIMATE [39] to calculate.

\section{NLRP3 mutations versus tumor microenvironment}

Overall infiltration of immune and stromal cells of each sample was evaluated with the aforementioned ESTIMATE algorithm. The nearest template prediction (NTP) algorithm [40] with a 48-gene signature [41] was applied to stratify melanoma patients into activated and normal stroma subgroups. A recent study reported that the activated stroma subtype exhibited an immunesuppressive role and a worse prognosis [41].

The infiltration abundance of 17 immune cell types was estimated with the CIBERSORT algorithm [42]. Angelova et al. established 812 immune metagene signatures to infer 31 immune cells infiltration and tumor immune landscape [43]. We used both methods to obtain comprehensive results and to validate each other.

An integrated list of 33 immune checkpoint genes was acquired from a recently published study [44]. We analyzed the distinct distributions of the above immune cells and checkpoint genes based on NLRP 3 mutational statuses. All analyses in this section were performed with gene expression data of 465 samples from TCGA.

\section{GSVA and GSEA}

Single sample gene set enrichment analysis (ssGSEA) function embedded in GSVA package (V1.36.1) [45] was utilized to calculate the enrichment of a specific gene set for each patient. Differential analysis according to NLRP3 mutational statuses was performed with $\mathrm{R}$ package DESeq2 (V1.28.1) [46], which manages sequencing expression data. The $t$ values obtained from differential analysis were subsequently used to conducted gene set enrichment analysis (GSEA) implemented by fgsea package (V1.14.0) (https://github.com/ctlab/fgsea). Kyoto encyclopedia of genes and genomes (KEGG) pathways were utilized as the background dataset.

\section{Statistical analyses}

$\mathrm{R}$ software (V4.0.1) and its affiliated packages were downloaded to complete related calculations and analyses. Waterfall plot of mutational patterns was achieved through GenVisR package (V1.20.0) [47]. Kaplan-Meier survival curve was drawn with survival (V2.41-3) and survminer (V0.4.7) packages and Logrank test to compare the difference. We used forestmodel package (V0.5.0) to perform multivariate regression analyses and to produce forest plots. Correlations of NLRP3 mutations with continuous and categorical factors were calculated with Wilcoxon ranksum test and Fisher exact test, separately.

\section{AUTHOR CONTRIBUTIONS}

SW designed this study; SW, QW, and JL developed the methodology and acquired the related data; QW, JL, WZ, FS, YR, QM, YL, TL, and XL performed data analysis and interpretation; QW, SW, and JL drafted and revised the manuscript; SW supervised this study. All authors read and approved the final manuscript.

\section{ACKNOWLEDGMENTS}

QHW would like to appreciate SZW at Weifang Medical University for her assistance in work and research. The authors thank Prof. Xiangchun $\mathrm{Li}$ at the Tianjin Medical University for the helpful suggestions.

\section{CONFLICTS OF INTEREST}

The authors declare that they have no conflicts of interest.

\section{FUNDING}

This study was supported by the Shandong Provincial Youth Innovation Team Development Plan of Colleges and Universities (No. 2019-6-156, Lu-Jiao), National Natural Science Foundation of China (No. 81872719 and 81803337), Provincial Natural Science Foundation of Shandong Province (No. ZR201807090257), and National Bureau of Statistics Foundation Project (No. 2018LY79).

\section{REFERENCES}

1. Roh W, Chen PL, Reuben A, Spencer CN, Prieto PA, Miller JP, Gopalakrishnan V, Wang F, Cooper ZA, Reddy SM, Gumbs C, Little L, Chang Q, et al. Integrated molecular analysis of tumor biopsies on sequential CTLA-4 and PD-1 blockade reveals markers of response and resistance. Sci Transl Med. 2017; 9:eaah3560. https://doi.org/10.1126/scitranslmed.aah3560 PMID:28251903

2. Twitty CG, Huppert LA, Daud AI. Prognostic Biomarkers for Melanoma Immunotherapy. Curr Oncol Rep. 2020; 22:25. https://doi.org/10.1007/s11912-020-0886-z PMID: $\underline{32048065}$

3. Ribas A, Wolchok JD. Cancer immunotherapy using checkpoint blockade. Science. 2018; 359:1350-5. https://doi.org/10.1126/science.aar4060 PMID:29567705 
4. Robert C, Grob JJ, Stroyakovskiy D, Karaszewska B, Hauschild A, Levchenko E, Chiarion Sileni V, Schachter J, Garbe C, Bondarenko I, Gogas H, Mandalá M, Haanen JB, et al. Five-Year Outcomes with Dabrafenib plus Trametinib in Metastatic Melanoma. N Engl J Med. 2019; 381:626-36.

https://doi.org/10.1056/NEJMoa1904059

PMID:31166680

5. Larkin J, Chiarion-Sileni V, Gonzalez R, Grob JJ, Cowey $C L$, Lao $C D$, Schadendorf $D$, Dummer $R$, Smylie $M$, Rutkowski P, Ferrucci PF, Hill A, Wagstaff J, et al. Combined Nivolumab and Ipilimumab or Monotherapy in Untreated Melanoma. N Engl J Med. 2015; 373: 23-34.

https://doi.org/10.1056/NEJMoa1504030 PMID:26027431

6. Havel JJ, Chowell D, Chan TA. The evolving landscape of biomarkers for checkpoint inhibitor immunotherapy. Nat Rev Cancer. 2019; 19:133-50.

https://doi.org/10.1038/s41568-019-0116-x PMID: $\underline{30755690}$

7. Yarchoan M, Hopkins A, Jaffee EM. Tumor Mutational Burden and Response Rate to PD-1 Inhibition. N Engl J Med. 2017; 377:2500-1.

https://doi.org/10.1056/NEJMc1713444 PMID:29262275

8. Samstein RM, Lee $\mathrm{CH}$, Shoushtari AN, Hellmann MD, Shen R, Janjigian YY, Barron DA, Zehir A, Jordan EJ, Omuro A, Kaley TJ, Kendall SM, Motzer RJ, et al. Tumor mutational load predicts survival after immunotherapy across multiple cancer types. Nat Genet. 2019; 51: 202-6.

https://doi.org/10.1038/s41588-018-0312-8

PMID:30643254

9. Chan TA, Yarchoan M, Jaffee E, Swanton C, Quezada SA, Stenzinger A, Peters S. Development of tumor mutation burden as an immunotherapy biomarker: utility for the oncology clinic. Ann Oncol. 2019; 30: 44-56.

https://doi.org/10.1093/annonc/mdy495 PMID:30395155

10. Sharma $P$, Callahan $M K$, Bono $P$, Kim J, Spiliopoulou $P$, Calvo E, Pillai RN, Ott PA, de Braud F, Morse M, Le DT, Jaeger D, Chan E, et al. Nivolumab monotherapy in recurrent metastatic urothelial carcinoma (CheckMate 032): a multicentre, open-label, two-stage, multi-arm, phase 1/2 trial. Lancet Oncol. 2016; 17:1590-8.

https://doi.org/10.1016/S1470-2045(16)30496-X

PMID:27733243

11. Mangan MS, Olhava EJ, Roush WR, Seidel HM, Glick GD, Latz E. Targeting the NLRP3 inflammasome in inflammatory diseases. Nat Rev Drug Discov. 2018; 17:588-606. https://doi.org/10.1038/nrd.2018.97

PMID: 30026524

12. Moossavi M, Parsamanesh N, Bahrami A, Atkin SL, Sahebkar A. Role of the NLRP3 inflammasome in cancer. Mol Cancer. 2018; 17:158.

https://doi.org/10.1186/s12943-018-0900-3

PMID: $\underline{30447690}$

13. Zaki $M H$, Boyd KL, Vogel $P$, Kastan MB, Lamkanfi $M$, Kanneganti TD. The NLRP3 inflammasome protects against loss of epithelial integrity and mortality during experimental colitis. Immunity. 2010; 32:379-91.

https://doi.org/10.1016/i.immuni.2010.03.003 PMID:20303296

14. Dupaul-Chicoine J, Yeretssian G, Doiron K, Bergstrom KS, Mclntire CR, LeBlanc PM, Meunier C, Turbide C, Gros P, Beauchemin N, Vallance BA, Saleh M. Control of intestinal homeostasis, colitis, and colitis-associated colorectal cancer by the inflammatory caspases. Immunity. 2010; 32:367-78.

https://doi.org/10.1016/i.immuni.2010.02.012 PMID:20226691

15. Li S, Liang X, Ma L, Shen L, Li T, Zheng L, Sun A, Shang W, Chen C, Zhao W, Jia J. MiR-22 sustains NLRP3 expression and attenuates $\mathrm{H}$. pylori-induced gastric carcinogenesis. Oncogene. 2018; 37:884-96. https://doi.org/10.1038/onc.2017.381 PMID:29059152

16. Fan SH, Wang YY, Lu J, Zheng YL, Wu DM, Li MQ, Hu B, Zhang ZF, Cheng W, Shan Q. Luteoloside suppresses proliferation and metastasis of hepatocellular carcinoma cells by inhibition of NLRP3 inflammasome. PLoS One. 2014; 9:e89961.

https://doi.org/10.1371/journal.pone.0089961 PMID:24587153

17. Bae JY, Lee SW, Shin YH, Lee JH, Jahng JW, Park K. P2X7 receptor and NLRP3 inflammasome activation in head and neck cancer. Oncotarget. 2017; 8:48972-82. https://doi.org/10.18632/oncotarget.16903 PMID:28430665

18. Sayan M, Mossman BT. The NLRP3 inflammasome in pathogenic particle and fibre-associated lung inflammation and diseases. Part Fibre Toxicol. 2016; 13:51.

https://doi.org/10.1186/s12989-016-0162-4 PMID:27650313

19. Veeranki S. Role of inflammasomes and their regulators in prostate cancer initiation, progression and metastasis. Cell Mol Biol Lett. 2013; 18:355-67. https://doi.org/10.2478/s11658-013-0095-y PMID:23793845

20. Li L, Liu Y. Aging-related gene signature regulated by Nlrp3 predicts glioma progression. Am J Cancer Res. 2014; 5:442-9.

PMID:25628952 
21. Ahmad I, Muneer KM, Tamimi IA, Chang ME, Ata MO, Yusuf $\mathrm{N}$. Thymoquinone suppresses metastasis of melanoma cells by inhibition of NLRP3 inflammasome. Toxicol Appl Pharmacol. 2013; 270:70-6. https://doi.org/10.1016/j.taap.2013.03.027 PMID:23583630

22. Guarda G, Zenger M, Yazdi AS, Schroder K, Ferrero I, Menu P, Tardivel A, Mattmann C, Tschopp J. Differential expression of NLRP3 among hematopoietic cells. J Immunol. 2011; 186:2529-34.

https://doi.org/10.4049/jimmunol.1002720 PMID:21257968

23. Drexler SK, Bonsignore L, Masin M, Tardivel A, Jackstadt R, Hermeking $H$, Schneider $P$, Gross $O$, Tschopp J, Yazdi AS. Tissue-specific opposing functions of the inflammasome adaptor ASC in the regulation of epithelial skin carcinogenesis. Proc Natl Acad Sci USA. 2012; 109:18384-9.

https://doi.org/10.1073/pnas.1209171109

PMID:23090995

24. Dunn JH, Ellis LZ, Fujita M. Inflammasomes as molecular mediators of inflammation and cancer: potential role in melanoma. Cancer Lett. 2012; 314: 24-33.

https://doi.org/10.1016/i.canlet.2011.10.001 PMID:22050907

25. van Deventer HW, Burgents JE, Wu QP, Woodford RM, Brickey WJ, Allen IC, McElvania-Tekippe E, Serody JS, Ting JP. The inflammasome component NLRP3 impairs antitumor vaccine by enhancing the accumulation of tumor-associated myeloid-derived suppressor cells. Cancer Res. 2010; 70:10161-9.

https://doi.org/10.1158/0008-5472.CAN-10-1921 PMID:21159638

26. Jia Q, Wang J, He N, He J, Zhu B. Titin mutation associated with responsiveness to checkpoint blockades in solid tumors. JCl Insight. 2019; 4:e127901.

https://doi.org/10.1172/jci.insight.127901 PMID:31092729

27. Wang $F$, Zhao Q, Wang YN, Jin Y, He MM, Liu ZX, Xu RH. Evaluation of POLE and POLD1 Mutations as Biomarkers for Immunotherapy Outcomes Across Multiple Cancer Types. JAMA Oncol. 2019; 5:1504-6. https://doi.org/10.1001/jamaoncol.2019.2963 PMID:31415061

28. Braun DA, Ishii Y, Walsh AM, Van Allen EM, Wu CJ, Shukla SA, Choueiri TK. Clinical Validation of PBRM1 Alterations as a Marker of Immune Checkpoint Inhibitor Response in Renal Cell Carcinoma. JAMA Oncol. 2019; 5:1631-3.

https://doi.org/10.1001/jamaoncol.2019.3158 PMID:31486842
29. Liu D, Schilling B, Liu D, Sucker A, Livingstone E, JerbyArnon L, Zimmer L, Gutzmer R, Satzger I, Loquai C, Grabbe S, Vokes N, Margolis CA, et al. Integrative molecular and clinical modeling of clinical outcomes to PD1 blockade in patients with metastatic melanoma. Nat Med. 2019; 25:1916-27. https://doi.org/10.1038/s41591-019-0654-5 PMID:31792460

30. Ferreira LM, Muller YD, Bluestone JA, Tang Q. Nextgeneration regulatory $T$ cell therapy. Nat Rev Drug Discov. 2019; 18:749-69. https://doi.org/10.1038/s41573-019-0041-4 PMID:31541224

31. Denton AE, Roberts EW, Fearon DT. Stromal Cells in the Tumor Microenvironment. Adv Exp Med Biol. 2018; 1060:99-114. https://doi.org/10.1007/978-3-319-78127-3 6 PMID: 30155624

32. Van Allen EM, Miao D, Schilling B, Shukla SA, Blank C, Zimmer L, Sucker A, Hillen U, Foppen $\mathrm{MH}$, Goldinger SM, Utikal J, Hassel JC, Weide B, et al. Genomic correlates of response to CTLA-4 blockade in metastatic melanoma. Science. 2015; 350:207-11. https://doi.org/10.1126/science.aad0095 PMID:26359337

33. Hugo W, Zaretsky JM, Sun L, Song C, Moreno BH, HuLieskovan S, Berent-Maoz B, Pang J, Chmielowski B, Cherry G, Seja E, Lomeli S, Kong X, et al. Genomic and Transcriptomic Features of Response to Anti-PD-1 Therapy in Metastatic Melanoma. Cell. 2016; 165: 35-44.

https://doi.org/10.1016/j.cell.2016.02.065 PMID:26997480

34. Riaz N, Havel JJ, Makarov V, Desrichard A, Urba WJ, Sims JS, Hodi FS, Martín-Algarra S, Mandal R, Sharfman WH, Bhatia S, Hwu WJ, Gajewski TF, et al. Tumor and Microenvironment Evolution during Immunotherapy with Nivolumab. Cell. 2017; 171:934-49.e16.

https://doi.org/10.1016/i.cell.2017.09.028 PMID:29033130

35. Snyder A, Makarov V, Merghoub T, Yuan J, Zaretsky JM, Desrichard A, Walsh LA, Postow MA, Wong P, Ho TS, Hollmann TJ, Bruggeman C, Kannan K, et al. Genetic basis for clinical response to CTLA-4 blockade in melanoma. N Engl J Med. 2014; 371:2189-99.

https://doi.org/10.1056/NEJMoa1406498 PMID:25409260

36. Zaretsky JM, Garcia-Diaz A, Shin DS, Escuin-Ordinas $H$, Hugo W, Hu-Lieskovan S, Torrejon DY, Abril-Rodriguez G, Sandoval S, Barthly L, Saco J, Homet Moreno B, Mezzadra R, et al. Mutations Associated with Acquired Resistance to PD-1 Blockade in Melanoma. N Engl J Med. 2016; 375:819-29. 
https://doi.org/10.1056/NEJMoa1604958

PMID:27433843

37. Ramos $A H$, Lichtenstein L, Gupta M, Lawrence MS, Pugh TJ, Saksena G, Meyerson M, Getz G. Oncotator: cancer variant annotation tool. Hum Mutat. 2015; 36:E2423-9.

https://doi.org/10.1002/humu.22771 PMID:25703262

38. Grzywa TM, Paskal W, Włodarski PK. Intratumor and Intertumor Heterogeneity in Melanoma. Transl Oncol. 2017; 10:956-75.

https://doi.org/10.1016/i.tranon.2017.09.007 PMID:29078205

39. Yoshihara K, Shahmoradgoli M, Martínez E, Vegesna R, Kim H, Torres-Garcia W, Treviño V, Shen H, Laird PW, Levine DA, Carter SL, Getz G, Stemke-Hale K, et al. Inferring tumour purity and stromal and immune cell admixture from expression data. Nat Commun. 2013; 4:2612.

https://doi.org/10.1038/ncomms3612 PMID:24113773

40. Hoshida Y. Nearest template prediction: a singlesample-based flexible class prediction with confidence assessment. PLoS One. 2010; 5:e15543.

https://doi.org/10.1371/journal.pone.0015543 PMID:21124904

41. Moffitt RA, Marayati R, Flate EL, Volmar KE, Loeza SG, Hoadley KA, Rashid NU, Williams LA, Eaton SC, Chung $\mathrm{AH}$, Smyla JK, Anderson JM, Kim HJ, et al. Virtual microdissection identifies distinct tumor- and stromaspecific subtypes of pancreatic ductal adenocarcinoma. Nat Genet. 2015; 47:1168-78.

https://doi.org/10.1038/ng.3398 PMID:26343385

42. Newman $A M$, Liu CL, Green $M R$, Gentles AJ, Feng W, Xu Y, Hoang $C D$, Diehn $M$, Alizadeh AA. Robust enumeration of cell subsets from tissue expression profiles. Nat Methods. 2015; 12:453-7.

https://doi.org/10.1038/nmeth.3337

PMID:25822800

43. Angelova $M$, Charoentong $P$, Hackl $H$, Fischer $M L$, Snajder R, Krogsdam AM, Waldner MJ, Bindea G, Mlecnik B, Galon J, Trajanoski Z. Characterization of the immunophenotypes and antigenomes of colorectal cancers reveals distinct tumor escape mechanisms and novel targets for immunotherapy. Genome Biol. 2015; 16:64.

https://doi.org/10.1186/s13059-015-0620-6

PMID:25853550

44. Ye $Y$, Jing $Y$, Li L, Mills GB, Diao L, Liu H, Han L. Sexassociated molecular differences for cancer immunotherapy. Nat Commun. 2020; 11:1779. https://doi.org/10.1038/s41467-020-15679-x PMID:32286310

45. Hänzelmann S, Castelo R, Guinney J. GSVA: gene set variation analysis for microarray and RNA-seq data. BMC Bioinformatics. 2013; 14:7. https://doi.org/10.1186/1471-2105-14-7 PMID:23323831

46. Love MI, Huber W, Anders S. Moderated estimation of fold change and dispersion for RNA-seq data with DESeq2. Genome Biol. 2014; 15:550. https://doi.org/10.1186/s13059-014-0550-8 PMID:25516281

47. Skidmore ZL, Wagner $A H$, Lesurf $R$, Campbell KM, Kunisaki J, Griffith OL, Griffith M. GenVisR: Genomic Visualizations in R. Bioinformatics. 2016; 32:3012-14. https://doi.org/10.1093/bioinformatics/btw325 PMID:27288499 


\section{SUPPLEMENTARY MATERIALS}

\section{Supplementary Figures}
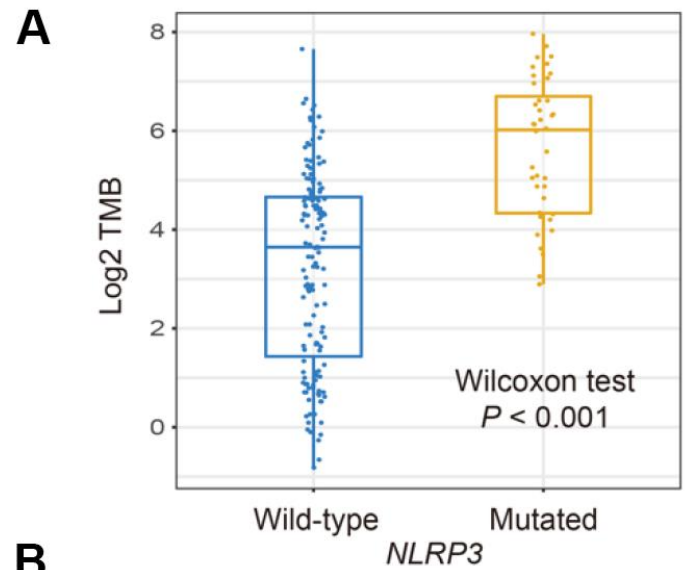

B

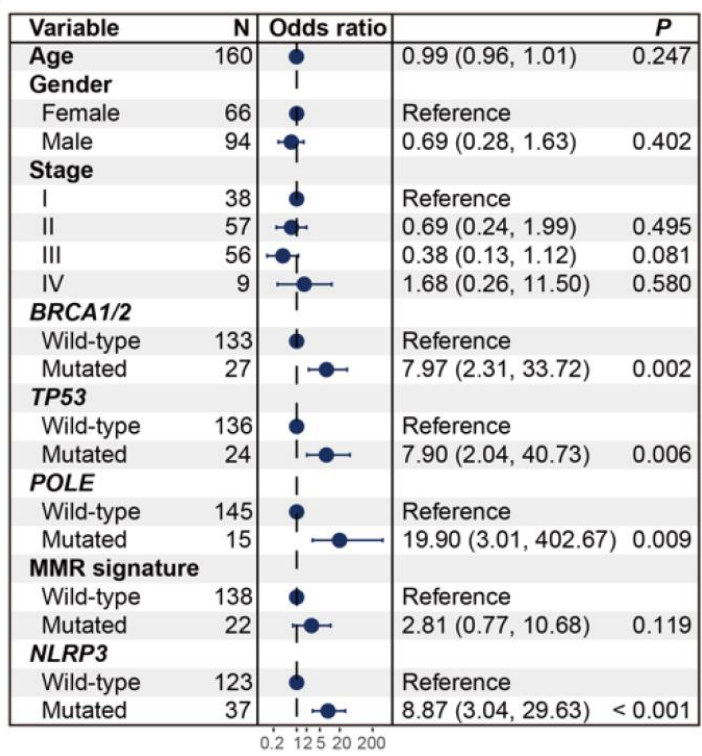

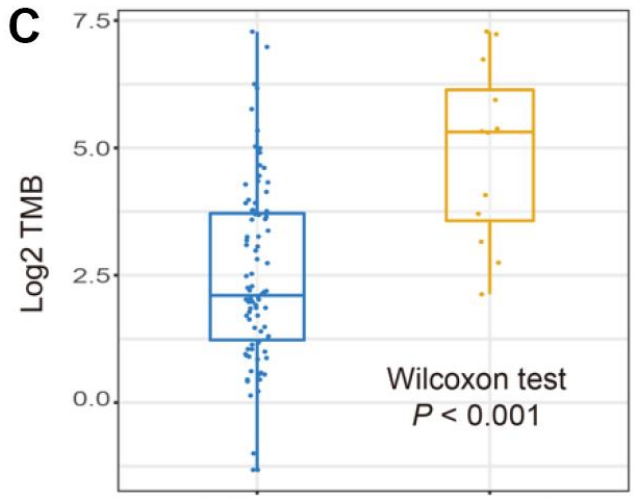

Wild-type Mutated NLRP3

D

\begin{tabular}{|c|c|c|c|c|}
\hline Variable & $\mathbf{N}$ & Odds ratio & & $P$ \\
\hline Age & 95 & $\boldsymbol{\sigma}$ & $1.03(0.99,1.09)$ & 0.196 \\
\hline \multicolumn{2}{|l|}{ Gender } & I & & \\
\hline Female & 40 & $\varphi$ & Reference & \\
\hline Male & 55 & & $1.21(0.26,5.76)$ & 0.807 \\
\hline \multicolumn{5}{|l|}{ Stage } \\
\hline I & 11 & b & Reference & \\
\hline II & 31 & + & $6.11(0.54,135.17)$ & 0.189 \\
\hline III & 32 & -9 & $0.55(0.02,16.31)$ & 0.720 \\
\hline IV & 21 & & $6.15(0.54,143.62)$ & 0.189 \\
\hline \multicolumn{5}{|l|}{ BRCA1/2 } \\
\hline Wild-type & 91 & 由 & Reference & \\
\hline Mutated & 4 & $T$ & $7.20(0.47,192.68)$ & 0.161 \\
\hline \multicolumn{5}{|l|}{ TP53 } \\
\hline Wild-type & 89 & 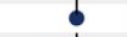 & Reference & \\
\hline Mutated & 6 & $-b$ & $0.93(0.02,30.15)$ & 0.970 \\
\hline \multicolumn{5}{|l|}{ POLE } \\
\hline Wild-type & 92 & & Reference & \\
\hline Mutated & 3 & & $1.25(0.05,58.10)$ & 0.893 \\
\hline \multicolumn{5}{|c|}{ MMR signature } \\
\hline Wild-type & 82 & $\bullet$ & Reference & \\
\hline Mutated & 13 & $1-$ & $40.14(6.25,457.92)$ & 0.001 \\
\hline \multicolumn{5}{|l|}{$N L R P 3$} \\
\hline Wild-type & 85 & d & Reference & \\
\hline Mutated & 10 & to- & $10.38(1.38,101.34)$ & 0.028 \\
\hline
\end{tabular}

Supplementary Figure 1. Association of NLRP3 mutations with TMB in 2 ICGC melanoma cohorts. NLRP3 mutations versus TMB with (A) univariate analysis and (B) multivariate regression model in MELA-AU cohort; NLRP3 mutations versus TMB with (C) univariate analysis and (D) multivariate regression model in SKCA-BR cohort. 


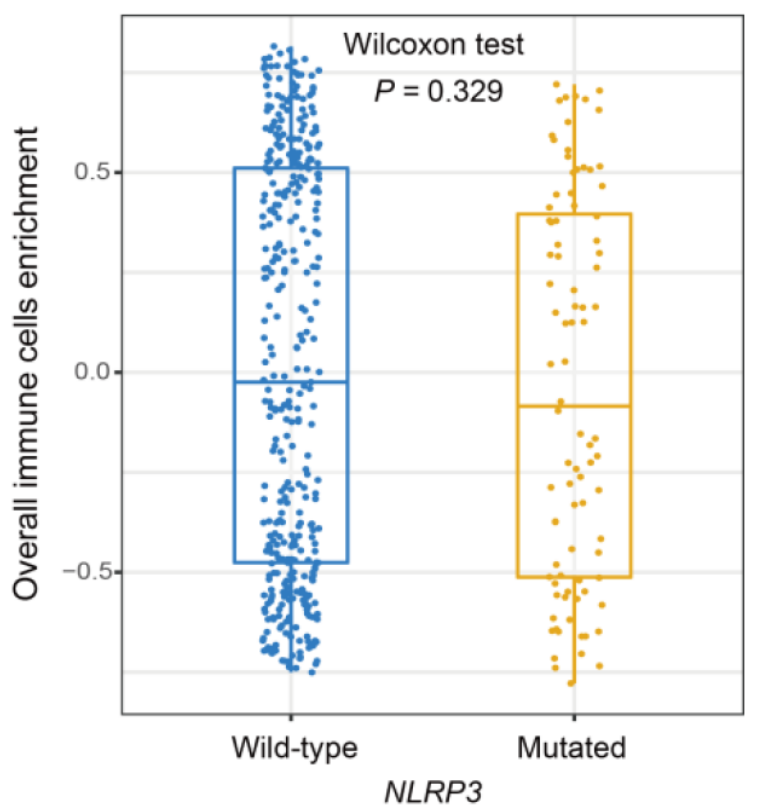

Supplementary Figure 2. Infiltration of overall immune cells in patients with and without NLRP3 mutations.

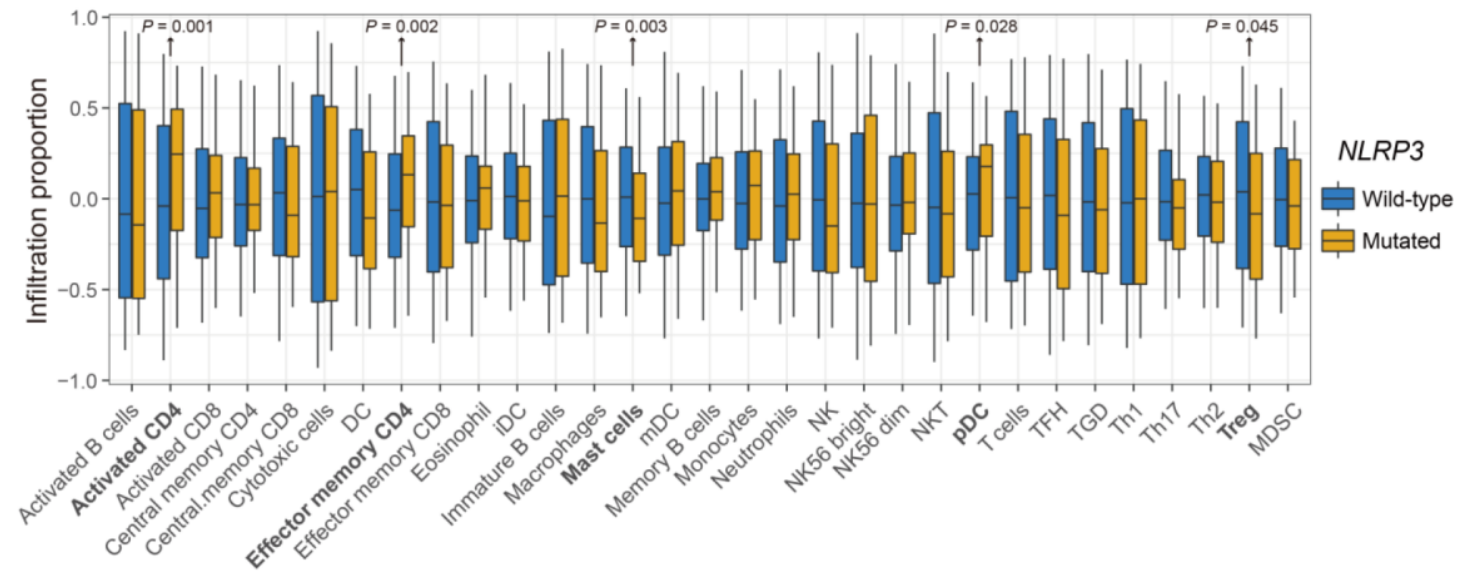

Supplementary Figure 3. Infiltration of $\mathbf{3 1}$ immune cells stratified by NLRP3 mutation status according to Angelova et al. method. 


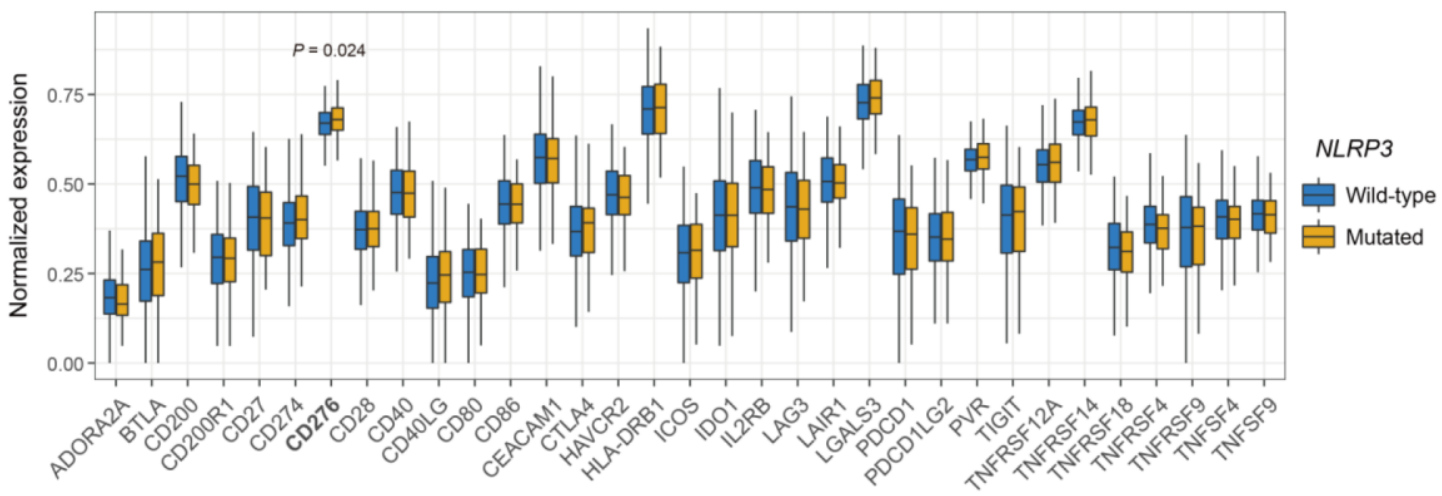

Supplementary Figure 4. Expression of 33 immune checkpoint genes stratified by NLRP3 mutation status.

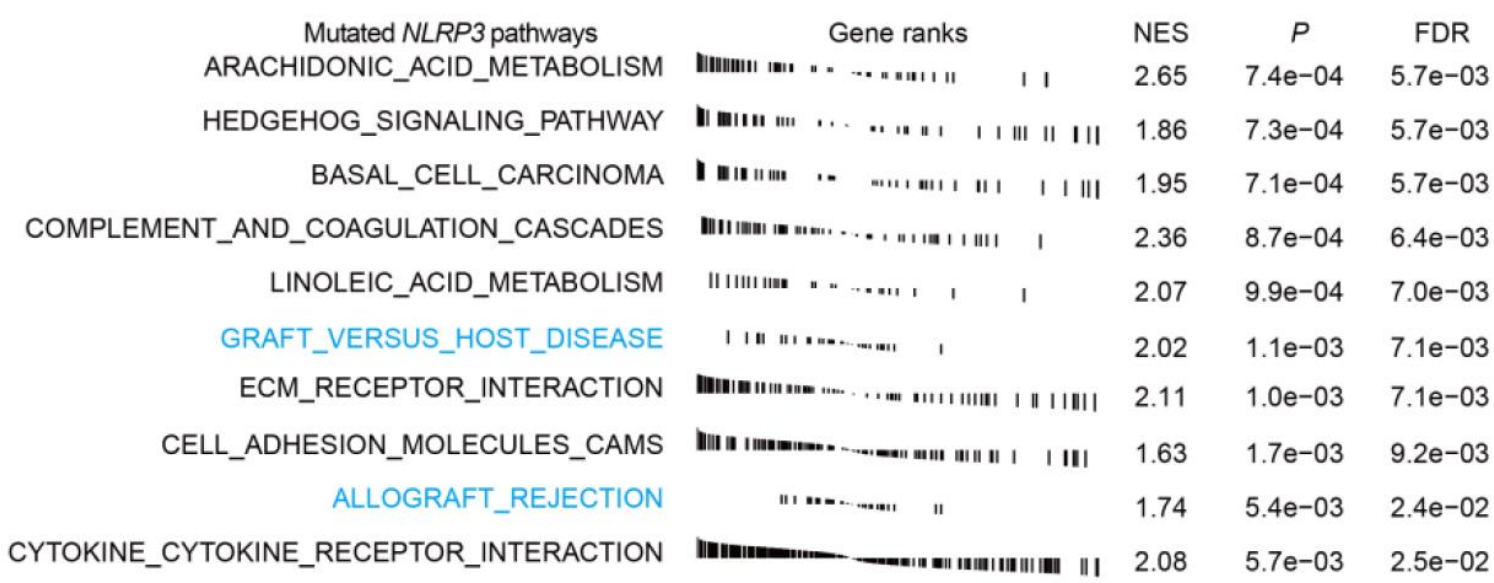

Supplementary Figure 5. GSEA analysis of NLRP3 mutant patients. 

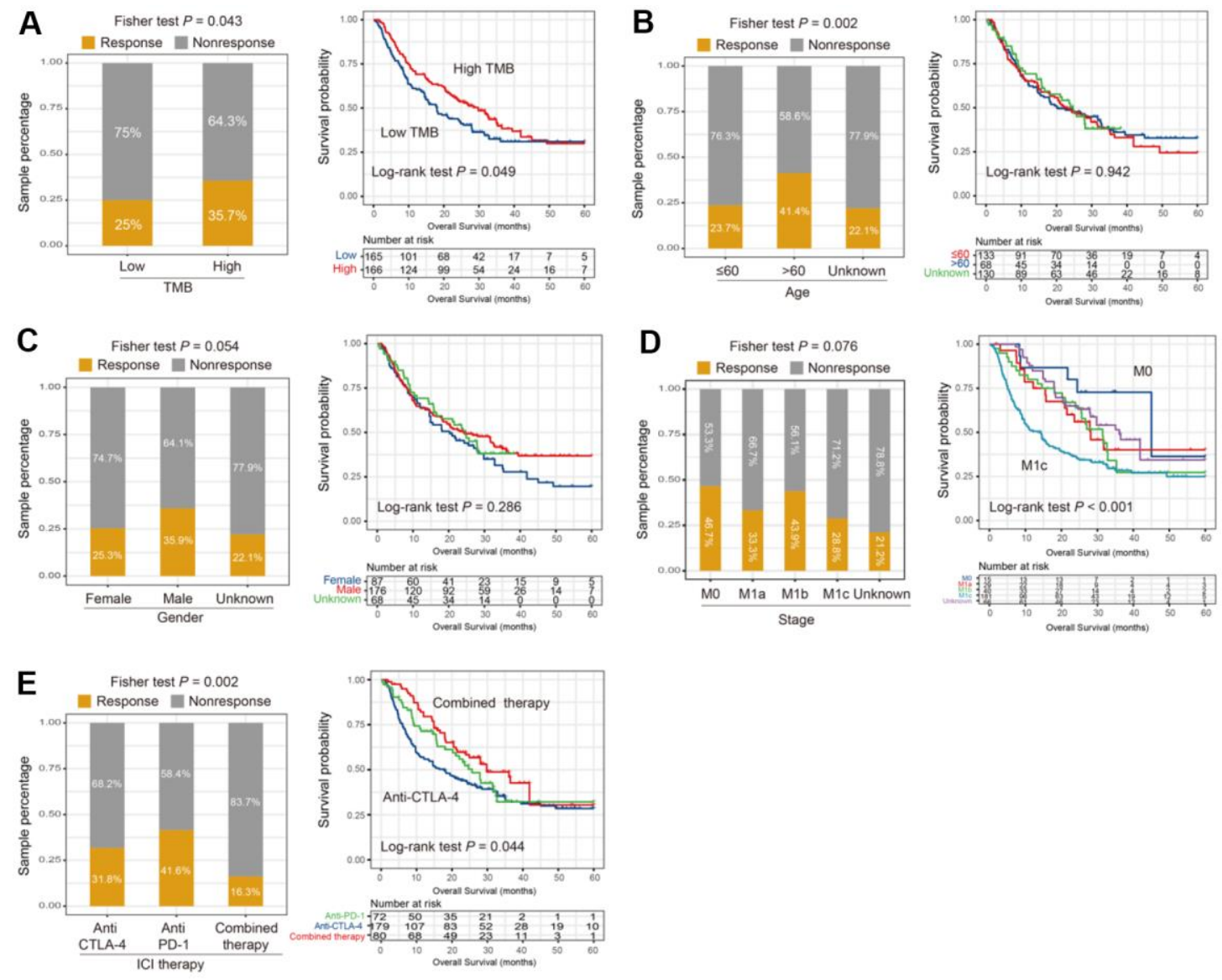

Supplementary Figure 6. Clinical characteristics association with ICl efficacy. Association of (A) TMB, (B) age, (C) gender, (D) stage, and (E) treatment type with $\mathrm{ICl}$ response rate and prognosis.
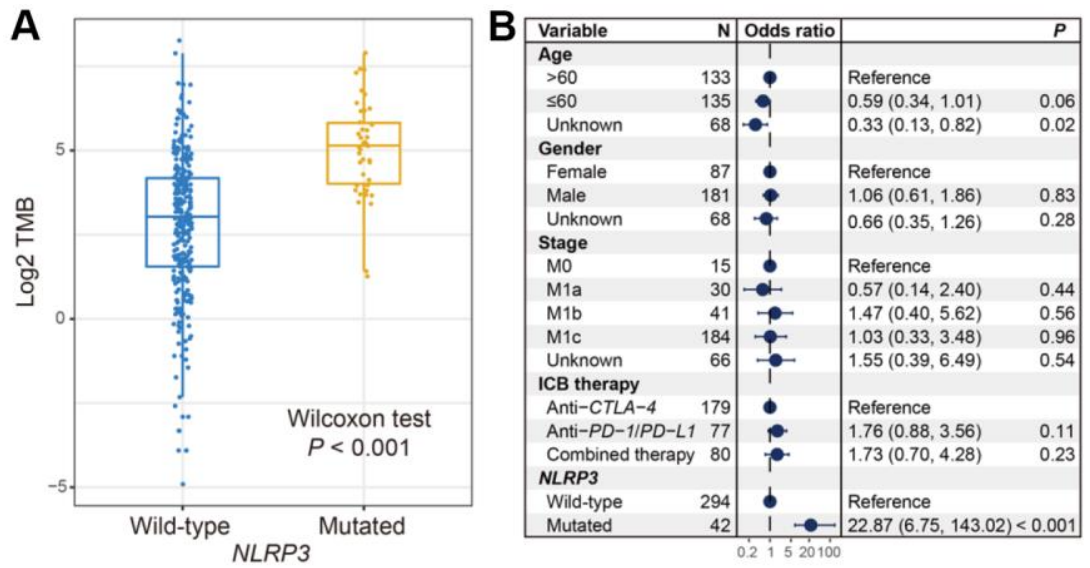

Supplementary Figure 7. Correlation of NLRP3 mutations with TMB in the pooled ICl-treated cohort. (A) Wilcoxon rank-sum test and (B) multivariate Logistic regression analysis for the correlation of NLRP3 mutations with TMB. 

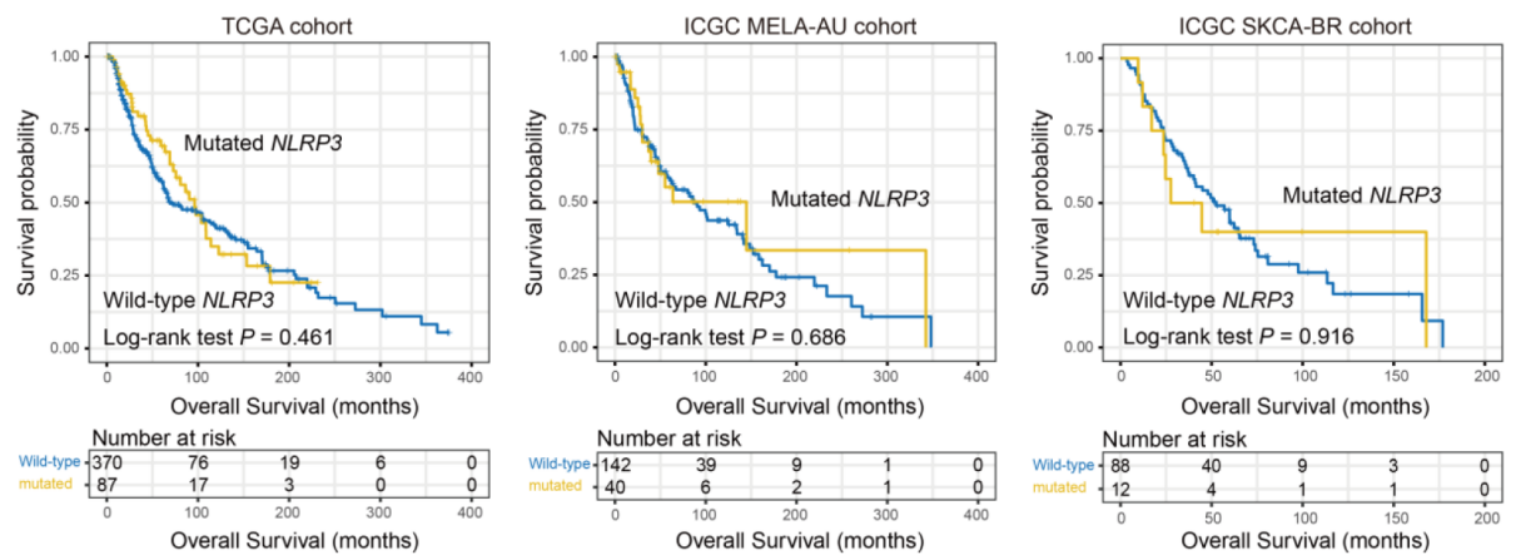

Supplementary Figure 8. Kaplan-Meier survival analysis of NLRP3 mutations in 3 non-ICl-treated cohorts.
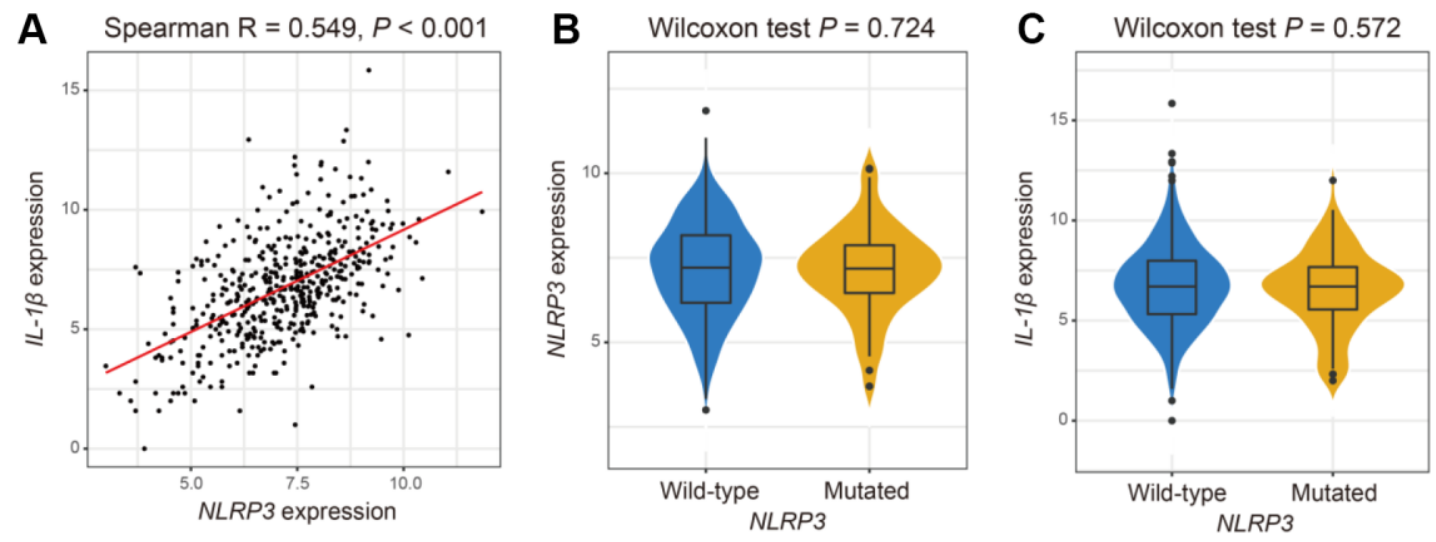

Supplementary Figure 9. NLRP3 and IL-1 $\beta$ mRNA expression concerning NLRP3 mutational status in melanoma based on the data from TCGA cohort. (A) Correlation analysis between NLRP3 and IL-1 $\beta$ expression. Distinct expression of (B) NLRP3 and (C) IL-1 $\beta$ in NLRP3 wild-type versus mutated subgroups. 


\section{Supplementary Tables}

Please browse Full Text version to see the data of Supplementary Table 2.

Supplementary Table 1. Association of NLRP3 mutations with clinical characteristics in 336 ICItreated melanoma patients.

\begin{tabular}{|c|c|c|c|c|}
\hline \multirow{2}{*}{ Characteristics } & \multirow{2}{*}{$n(\%)(N=366)$} & \multicolumn{2}{|c|}{ NLRP3 } & \multirow{2}{*}{$P$} \\
\hline & & Wild-type (n = 294) & Mutated $(n=42)$ & \\
\hline \multicolumn{5}{|l|}{ Age, years } \\
\hline$\leq 60$ & $135(40.2 \%)$ & $122(41.5 \%)$ & $13(30.9 \%)$ & \multirow{3}{*}{0.112} \\
\hline$>60$ & $133(39.6 \%)$ & $110(37.4 \%)$ & $23(54.8 \%)$ & \\
\hline Unknown & $68(20.2 \%)$ & $62(21.1 \%)$ & $6(14.3 \%)$ & \\
\hline \multicolumn{5}{|l|}{ Gender } \\
\hline Male & $181(53.9 \%)$ & $152(51.7 \%)$ & $29(69.0 \%)$ & \multirow{3}{*}{0.119} \\
\hline Female & $87(25.9 \%)$ & $80(27.2 \%)$ & $7(16.7 \%)$ & \\
\hline Unknown & $68(20.2 \%)$ & $62(21.1 \%)$ & $6(14.3 \%)$ & \\
\hline \multicolumn{5}{|l|}{ Stage } \\
\hline M0 & $15(4.5 \%)$ & $13(4.4 \%)$ & $2(4.8 \%)$ & \multirow{5}{*}{0.343} \\
\hline M1a & $30(8.9 \%)$ & $29(9.9 \%)$ & $1(2.4 \%)$ & \\
\hline M1b & $41(12.2 \%)$ & $36(12.2 \%)$ & $5(11.8 \%)$ & \\
\hline M1c & $184(54.8 \%)$ & $162(55.1 \%)$ & $22(52.4 \%)$ & \\
\hline Unknown & $66(19.6 \%)$ & $54(18.4 \%)$ & $12(28.6 \%)$ & \\
\hline \multicolumn{5}{|l|}{ ICB treatment } \\
\hline Anti-CTLA-4 & $179(53.3 \%)$ & $158(53.7 \%)$ & $21(50.0 \%)$ & \multirow{3}{*}{0.882} \\
\hline Anti-PD-1 & $77(22.9 \%)$ & $67(22.8 \%)$ & $10(23.8 \%)$ & \\
\hline Combined therapy & $80(23.8 \%)$ & $69(23.5 \%)$ & $11(26.2 \%)$ & \\
\hline \multicolumn{5}{|l|}{ RECIST } \\
\hline $\mathrm{CR} / \mathrm{PR}$ & $102(30.4 \%)$ & $83(28.2 \%)$ & $19(45.2 \%)$ & \multirow{3}{*}{0.229} \\
\hline SD & $43(12.8 \%)$ & $39(13.3 \%)$ & $4(9.5 \%)$ & \\
\hline PD & $191(56.8 \%)$ & $172(58.5 \%)$ & $19(45.3 \%)$ & \\
\hline \multicolumn{5}{|l|}{ OS status } \\
\hline Alive & $132(39.3 \%)$ & $108(36.7 \%)$ & $24(57.1 \%)$ & \multirow{3}{*}{0.002} \\
\hline Dead & $200(59.5 \%)$ & $184(62.6 \%)$ & $16(38.1 \%)$ & \\
\hline Unknown & $4(1.2 \%)$ & $2(0.7 \%)$ & $2(4.8 \%)$ & \\
\hline \multicolumn{5}{|l|}{$\mathrm{TMB}$} \\
\hline Low & $168(50.0 \%)$ & $166(56.5 \%)$ & $2(4.8 \%)$ & \multirow{2}{*}{$<0.001$} \\
\hline High & $168(50.0 \%)$ & $128(43.5)$ & $40(95.2 \%)$ & \\
\hline
\end{tabular}

Supplementary Table 2. Clinical characteristics of included $336 \mathrm{ICl}$-treated melanoma patients. 